\title{
Virial expansion for a strongly correlated Fermi gas with imbalanced spin populations
}

\author{
Xia-Ji Liu四 and Hui Hu周 \\ ${ }^{1}$ ARC Centre of Excellence for Quantum-Atom Optics, \\ Centre for Atom Optics and Ultrafast Spectroscopy, \\ Swinburne University of Technology, Melbourne 3122, Australia
}

(Dated: October 8, 2018)

\begin{abstract}
Quantum virial expansion provides an ideal tool to investigate the high-temperature properties of a strongly correlated Fermi gas. Here, we construct the virial expansion in the presence of spin population imbalance. Up to the third order, we calculate the high-temperature free energy of a unitary Fermi gas as a function of spin imbalance, with infinitely large, attractive or repulsive interactions. In the latter repulsive case, we show that there is no itinerant ferromagnetism when quantum virial expansion is applicable. We therefore estimate an upper bound for the ferromagnetic transition temperature $T_{c}$. For a harmonically trapped Fermi gas at unitarity, we find that $\left(T_{c}\right)_{\text {uppper }}<T_{F}$, where $T_{F}$ is the Fermi temperature at the center of the trap. Our result for the high-temperature equations of state may confront future high-precision thermodynamic measurements.
\end{abstract}

\section{INTRODUCTION}

Strongly interacting Fermi gases of ultracold atoms near Feshbach resonances have offered unique opportunity to study some long-sought problems in condensed matter physics, astrophysics and particle physics [1, 2]. An particular example that attracts intense interests is the ground state of a two-component, spin-population imbalanced Fermi gas of ${ }^{6} \mathrm{Li}$ or ${ }^{40} \mathrm{~K}$ atoms [3, 4]. In the strongly interacting regime, observation of new exotic superfluid states in the imbalanced systems may be anticipated, such as the spatially inhomogeneous FuldeFerrell-Larkin-Ovchinnikov state [5-12]. However, because of strong interactions, theoretical investigation of imbalanced Fermi systems turns out to be difficult and restrictive.

In this work, we theoretically investigate the hightemperature equation of state of a strongly interacting, imbalanced Fermi gas, by generalizing a quantum virial expansion method to imbalanced systems $13-16,18,19]$. Virial expansion for balanced Fermi gases with equal spin populations has already shown to be very useful, in the studies of both static [13-17] and dynamic properties [18, 19]. The third virial coefficient of a unitary Fermi gas with infinitely large scattering length has been calculated [15] and consequently confirmed experimentally [20]. The third virial coefficient of strongly interacting Bose gases has been determined 21]. The virial expansion of singleparticle spectral function [18] or dynamic structure factor [19] has also been developed to understand, respectively, the relevant measurements of momentum-resolved rf spectroscopy [22, 23] or Bragg spectroscopy [24].

Our generalization of quantum virial expansion to the imbalanced systems gives an approximate means to study interesting phenomena associated with the spin degree of freedom. For instance, for a strongly repulsively in-

\footnotetext{
*Electronic address: xiajiliu@swin.edu.au
}

${ }^{\dagger}$ Electronic address: hhu@swin.edu.au teracting Fermi gas, itinerant ferromagnetic transition is expected at low temperatures [25-30]. However, the critical transition temperature is yet to be determined. The use of virial expansion may allow us to estimate an upper bound for the critical temperature. By expanding to the third order, we find that there is no itinerant ferromagnetism as far as the quantum virial expansion is applicable. Therefore, we show that for a strongly repulsive Fermi gas in harmonic traps, the critical temperature should be smaller than the Fermi temperature at the center of the trap. We note that, virial expansion method has been used by Horowitz and Schwenk to study the low density, spin-imbalanced neutron matter [31, 32]. Their expansion was taken up to the second order, where the second virial coefficient was calculated from the nucleonnucleon scattering phase shifts.

Our paper is organized as follows. In the next section, we show how to expand the thermodynamic potential (or pressure in case of a uniform system) of an imbalanced Fermi system in terms of virial coefficients. The $n$-th virial coefficients can be calculated from the partition functions of a cluster that contains up to $n$-particles. Different spin configurations in a $n$-particle cluster give rise to different virial coefficients. In Sec. III, we focus on the expansion to the third order and calculate the finite temperature free energy of an imbalanced Fermi gas as a function of spin imbalance. The possibility of itinerant ferromagnetism in a strongly repulsively interacting system is discussed. Sec. V is devoted to conclusions and some final remarks.

\section{QUANTUM VIRIAL EXPANSION FOR AN IMBALANCED FERMI GAS}

The starting point of quantum virial expansion is that at large temperatures the chemical potential $\mu$ diverges to $-\infty$. Therefore, the fugacity $z \equiv \exp \left(\mu / k_{B} T\right) \equiv$ $\exp (\beta \mu) \ll 1$ is a well-defined small expansion parameter. We may expand the high-temperature thermodynamic potential $\Omega$ of a quantum system in powers of fugacity, 
no matter how strong the interaction strength would be.

For a two-component Fermi gas with balanced spin populations, it was shown that [15, 16],

$$
\Omega=-k_{B} T Q_{1}\left[z+b_{2} z^{2}+\cdots+b_{n} z^{n}+\cdots\right],
$$

where $Q_{n}=\operatorname{Tr}_{n}\left[\exp \left(-\mathcal{H} / k_{B} T\right)\right]$ is the partition function of a cluster containing $n$ particles and $b_{n}$ is the $n$-th virial expansion coefficient. The trace $\operatorname{Tr}_{n}$ takes into account all the spin-configurations of $n$-particles and traces over all the states of a proper symmetry. The virial coefficient $b_{n}$ was found to take the form [15, 16],

$$
b_{2}=Q_{2} / Q_{1}-Q_{1} / 2
$$

and

$$
b_{3}=Q_{3} / Q_{1}-Q_{2}+Q_{1}^{2} / 3, \quad \text { etc. }
$$

In the presence of spin imbalance, it is necessary to introduce two fugacities $z_{\uparrow} \equiv \exp \left(\mu_{\uparrow} / k_{B} T\right)$ and $z_{\downarrow} \equiv \exp \left(\mu_{\downarrow} / k_{B} T\right)$, and to distinguish different spinconfigurations. Quite generally, we may write the thermodynamic potential as,

$$
\Omega=-k_{B} T Q_{1} \sum_{n=1}^{\infty} \sum_{k=0}^{n} z_{\uparrow}^{n-k} z_{\downarrow}^{k} b_{n, k},
$$

where $b_{n, k}$ is the $n$-th (imbalanced) virial coefficient contributed by the configuration with $n-k$ spin-up fermions and $k$ spin-down fermions. It is easy to see that the imbalanced virial coefficients satisfy the relation $b_{n, k}=$ $b_{n, n-k}$ and $\sum_{k=0}^{n} b_{n, k}=b_{n}$.

The calculation of $b_{n, k}$ is straightforward, following the standard definition of thermodynamic potential. We rewrite the grand partition function $\mathcal{Z} \equiv \operatorname{Tr} \exp [-(\mathcal{H}-$ $\left.\left.\mu_{\uparrow} \mathcal{N}_{\uparrow}-\mu_{\downarrow} \mathcal{N}_{\downarrow}\right) / k_{B} T\right]$ in the form,

$$
\mathcal{Z}=\sum_{n=0}^{\infty} \sum_{k=0}^{n} z_{\uparrow}^{n-k} z_{\downarrow}^{k} Q_{n, k},
$$

where $Q_{n, k}$ is the partition function of a cluster that contains $n-k$ spin-up fermions and $k$ spin-down fermions. It is apparent that due to the symmetry in spin configurations we have $Q_{n, k}=Q_{n, n-k}$. The imbalanced cluster partition functions satisfy as well a sum rule $\sum_{k=0}^{n} Q_{n, k}=Q_{n}$. By expanding the thermodynamic potential $\Omega=-k_{B} T \ln \mathcal{Z}$ into powers of the two fugacities, the imbalanced virial coefficients can then be expressed in terms of the cluster partition function $Q_{n, k}$.

\section{A. Virial expansion up to the third order}

To be concrete, let us consider the imbalanced virial expansion up to the third order. To this order, we may write the grand partition function as $\mathcal{Z}=1+x_{1}+x_{2}+x_{3}$, where

$$
x_{1}=z_{\uparrow} Q_{1,0}+z_{\downarrow} Q_{1,1}
$$

$$
x_{2}=z_{\uparrow}^{2} Q_{2,0}+z_{\uparrow} z_{\downarrow} Q_{2,1}+z_{\downarrow}^{2} Q_{2,2},
$$

and

$$
x_{3}=z_{\uparrow}^{3} Q_{3,0}+z_{\uparrow}^{2} z_{\downarrow} Q_{3,1}+z_{\uparrow} z_{\downarrow}^{2} Q_{3,2}+z_{\downarrow}^{3} Q_{3,3} .
$$

By introducing a symmetric cluster partition function $Q_{n}^{s} \equiv Q_{n, 0}=Q_{n, n}$ and using the properties of $Q_{n, k}$, it is easy to show that $Q_{1}^{s}=Q_{1} / 2, Q_{2,1}=Q_{2}-2 Q_{2}^{s}$, and $Q_{3,1}=Q_{3,2}=Q_{3} / 2-Q_{3}^{s}$. Using $\ln \left(1+x_{1}+x_{2}+x_{3}\right) \simeq$ $\left(x_{1}+x_{2}+x_{3}\right)-\left(x_{1}^{2}+2 x_{1} x_{2}\right) / 2+x_{1}^{3} / 3$, after some algebra we obtain $b_{n, k}(k \leq n / 2)$,

$$
b_{1,0}=1 / 2
$$

$$
b_{2,0}=Q_{2}^{s} / Q_{1}-Q_{1} / 8
$$

$$
\begin{gathered}
b_{2,1}=Q_{2} / Q_{1}-2 Q_{2}^{s} / Q_{1}-Q_{1} / 4, \\
b_{3,0}=Q_{3}^{s} / Q_{1}-Q_{2}^{s} / 2+Q_{1}^{2} / 24,
\end{gathered}
$$

and

$$
b_{3,1}=Q_{3} /\left(2 Q_{1}\right)-Q_{3}^{s} / Q_{1}-Q_{2} / 2+Q_{2}^{s} / 2+Q_{1}^{2} / 8 .
$$

The virial coefficients with $k \geq n / 2$ can be obtained directly since $b_{n, k}=b_{n, n-k}$.

In practice, it is convenient to consider the interaction effect on the virial coefficients or the differences such as $\Delta Q_{n}=Q_{n}-Q_{n}^{(1)}, \Delta b_{n}=b_{n}-b_{n}^{(1)}$, and $\Delta b_{n, k}=b_{n, k}-b_{n, k}^{(1)}$. Here, the superscript " 1 " denotes an ideal, non-interacting system with the same fugacities and the operator " $\Delta$ " removes the non-interacting contribution. It is clear that the symmetric cluster partition function $Q_{n}^{s}$ is not affected by interactions since the interatomic interaction occurs only between fermions with unlike spins. Thus, we have $\Delta b_{2,0}=\Delta b_{3,0}=0, \Delta b_{2,1}=$ $\Delta\left(b_{2}-2 b_{2,0}\right)=\Delta b_{2}$ and $\Delta b_{3,1}=\Delta\left(b_{3} / 2-b_{2,0}\right)=\Delta b_{3} / 2$. Accordingly, we may rewrite the thermodynamic potential into the form (up to the third order),

$$
\Omega=\Omega^{(1)}-k_{B} T Q_{1}\left[z_{\uparrow} z_{\downarrow} \Delta b_{2}+\frac{z_{\uparrow}^{2} z_{\downarrow}+z_{\uparrow} z_{\downarrow}^{2}}{2} \Delta b_{3}\right] ，
$$

where $\Omega^{(1)}$ is the thermodynamic potential of a noninteracting Fermi gas.

\section{B. Thermodynamic potential $\Omega^{(1)}$}

The thermodynamic potential of an ideal, imbalanced Fermi gas is simply the sum of thermodynamic potential of each component, $\Omega^{(1)}=\Omega^{(1)}\left(\mu_{\uparrow}\right)+\Omega^{(1)}\left(\mu_{\downarrow}\right)$. In homogeneous space, where $Q_{1, H}=2 V / \lambda^{3}$, the singlecomponent thermodynamic potential $\Omega_{H}^{(1)}\left(\mu_{\sigma}\right)$ takes the form $(\sigma=\uparrow, \downarrow)$,

$$
\Omega_{H}^{(1)}=-V \frac{k_{B} T}{\lambda^{3}} \frac{2}{\sqrt{\pi}} \int_{0}^{\infty} t^{1 / 2} \ln \left(1+z_{\sigma} e^{-t}\right) d t,
$$


where $V$ is the volume and $\lambda \equiv\left[2 \pi \hbar^{2} /\left(m k_{B} T\right)\right]^{1 / 2}$ is the thermal wavelength. We have used the subscript " $H$ " to denote the result in the homogeneous space.

In the presence of a harmonic trap $V_{T}(r)=m \omega^{2} r^{2} / 2$, we consider the thermodynamic limit with a large number of fermions. The non-interacting thermodynamic potential $\Omega_{T}^{(1)}\left(\mu_{\sigma}\right)$ is then given semiclassically by $(\beta \equiv$ $\left.1 / k_{B} T\right)$

$$
\begin{aligned}
\Omega_{T}^{(1)} & =-\frac{1}{\beta} \int \frac{d \mathbf{r} d \mathbf{k}}{(2 \pi)^{3}} \ln \left[1+e^{-\beta\left(\frac{\hbar^{2} k^{2}}{2 m}+\frac{m}{2} \omega^{2} r^{2}-\mu_{\sigma}\right)}\right](13) \\
& =-\frac{\left(k_{B} T\right)^{4}}{(\hbar \omega)^{3}} \frac{1}{2} \int_{0}^{\infty} t^{2} \ln \left(1+z_{\sigma} e^{-t}\right) d t
\end{aligned}
$$

Accordingly, we have $Q_{1, T}=2\left(k_{B} T\right)^{3} /(\hbar \omega)^{3}$. Here, the subscript " $T$ " denotes the result in a harmonic trap.

\section{Virial coefficients $\Delta b_{2}$ and $\Delta b_{3}$}

The calculation of virial coefficients requires the knowledge of full energy spectrum of a few-body system. By utilizing the exact two- and three-fermion solutions, we have calculated the second and third virial coefficients for a strongly attractively or repulsively interacting Fermi gas [16]. Here, the terminology of "a strongly attractively interacting Fermi gas" refers to a resonant Fermi gas near a Feshbach resonance with the inclusion of the molecular states, while the terminology of "a strongly repulsively inteacting Fermi gas" means a resonant Fermi gas with the molecular branch excluded. In other words, all fermions in the strongly repulsively interacting Fermi gas populate only on the upper, atomic branch of the energy levels near a Feshbach resonance. At unitarity, three resonantly interacting fermions in an isotropic harmonic trap can be solved exactly using hyperspherical coordinates [33, 34], with the relative energy given by $E_{r e l}=\left(2 q+s_{l, n}+1\right) \hbar \omega$, where $q$ and $s_{l, n}$ ( $l$ the relative angular momentum and $n$ the number of nodes) are respectively the good quantum numbers in the hyperradius and hyperangle equations. One can show that the molecular branch corresponds to the states with $s_{l, n=0}$ [16]. These states should be excluded for the "repulsive" case. We refer to the Sec. IIIB of Ref. [16] for a detailed discussion about this.

In the following, we shall focus on the unitarity limit with infinitely large scattering length, where the virial coefficients are universal and temperature independent. The coefficients for a homogeneous gas are given by [16],

$$
\begin{aligned}
& \Delta b_{2, H}^{a t t}=+1 / \sqrt{2} \\
& \Delta b_{2, H}^{r e p}=-1 / \sqrt{2}
\end{aligned}
$$

and

$$
\begin{aligned}
\Delta b_{3, H}^{a t t} & \simeq-0.35501298 \\
\Delta b_{3, H}^{r e p} & \simeq+1.8174
\end{aligned}
$$

Here, the superscript "att" (or "rep") means the coefficient of an attractively (or repulsively) interacting Fermi gas. The third virial coefficient of an attractive unitary Fermi gas, $\Delta b_{3, H}^{a t t} \simeq-0.355$, was recently confirmed experimentally at École Normale Supérieure (ENS), Paris [20].

For a trapped Fermi gas, we instead have,

$$
\begin{aligned}
& \Delta b_{2, T}^{a t t}=+1 / 4, \\
& \Delta b_{2, T}^{r e p}=-1 / 4,
\end{aligned}
$$

and

$$
\begin{aligned}
\Delta b_{3, T}^{a t t} & \simeq-0.06833960, \\
\Delta b_{3, T}^{r e p} & \simeq 0.34976 .
\end{aligned}
$$

We note that, in the unitarity limit the homogeneous and trapped virial coefficients are connected universally by the relation [15, 16],

$$
b_{n, T}=\frac{b_{n, H}}{n^{3 / 2}} .
$$

The factor of $n^{-3 / 2}$ reduction in harmonic traps is simply due to the higher density of states in traps. To see this, let us consider the thermodynamic potential of a harmonic trapped Fermi gas in the local density approximation $\Omega=\int d \mathbf{r} \Omega(\mathbf{r})$, where $\Omega(\mathbf{r})$ is the local thermodynamic potential

$$
\Omega(\mathbf{r}) \propto z(\mathbf{r})+b_{2, H} z^{2}(\mathbf{r})+b_{3, H} z^{3}(\mathbf{r})+\cdots .
$$

Here, the local fugacity $z(\mathbf{r})=z \exp \left[-V(\mathbf{r}) / k_{B} T\right]$ is determined by the local chemical potential $\mu(\mathbf{r})=\mu-V(\mathbf{r})$. The spatial integration immediately leads to Eq. (23). It is obivious that the suppressed virial coefficients in harmonic traps imply a weaker interaction effect and consequently a better convergence for virial expansion.

\section{Virial expansion of high- $T$ free energy}

We are now ready to study the high temperature thermodynamics of an imbalanced, strongly interacting Fermi gas. With the virial expansion of thermodynamic potential Eq. (11), we solve the standard thermodynamic relations $N_{\uparrow}=-\partial \Omega / \partial \mu_{\uparrow}$ and $N_{\downarrow}=-\partial \Omega / \partial \mu_{\downarrow}$ for the two fugacities $z_{\uparrow}$ and $z_{\downarrow}$, at a given reduced temperature $\tau=T / T_{F}$ and a given spin imbalance $P=\left(N_{\uparrow}-N_{\downarrow}\right) / N$. Here, $T_{F}$ is the Fermi temperature. It is given by $T_{F}=\hbar^{2}\left(3 \pi^{2} n\right)^{2 / 3} /(2 m) / k_{B}$ in the homogeneous space and by $T_{F}=(3 N)^{1 / 3} \hbar \omega / k_{B}$ in a harmonic trap.

In the homogeneous space, it is easy to show that we can define a dimensionless number density $\tilde{n}=n \lambda^{3}=$ $8 /\left(3 \sqrt{\pi} \tau^{3 / 2}\right), \tilde{n}_{\uparrow}=(1+p) \tilde{n} / 2$, and $\tilde{n}_{\downarrow}=(1-p) \tilde{n} / 2$. We then rewrite the number equations into dimensionless forms,

$$
\begin{aligned}
& \tilde{n}_{\uparrow}=n\left(z_{\uparrow}\right)+z_{\uparrow} z_{\downarrow} 2 \Delta b_{2}+\left(2 z_{\uparrow}^{2} z_{\downarrow}+z_{\uparrow} z_{\downarrow}^{2}\right) \Delta b_{3}, \\
& \tilde{n}_{\downarrow}=n\left(z_{\downarrow}\right)+z_{\uparrow} z_{\downarrow} 2 \Delta b_{2}+\left(z_{\uparrow}^{2} z_{\downarrow}+2 z_{\uparrow} z_{\downarrow}^{2}\right) \Delta b_{3},
\end{aligned}
$$


where $n(z) \equiv(2 / \sqrt{\pi}) \int_{0}^{\infty} \sqrt{t}\left[z e^{-t} /\left(1+z e^{-t}\right)\right] d t$. We can obtain the two fugacities by solving the coupled number equations. Then, we calculate the free energy $F=\Omega+\mu_{\uparrow} N_{\uparrow}+\mu_{\downarrow} N_{\downarrow}$. The free energy per particle in units of Fermi energy $E_{F}$ is given by,

$$
\begin{aligned}
\frac{F}{N E_{F}}= & \tau\left[\frac{1+p}{2} \ln z_{\uparrow}+\frac{1-p}{2} \ln z_{\downarrow}\right]-A\left[f\left(z_{\uparrow}\right)\right. \\
& +f\left(z_{\downarrow}\right)+z_{\uparrow} z_{\downarrow} 2 \Delta b_{2}+\left(z_{\uparrow}^{2} z_{\downarrow}+z_{\uparrow} z_{\downarrow}^{2}\right) \Delta b_{3}(27)
\end{aligned}
$$

where $f(z) \equiv(2 / \sqrt{\pi}) \int_{0}^{\infty} \sqrt{t} \ln \left(1+z e^{-t}\right) d t$ and the constant $A=3 \sqrt{\pi} \tau^{5 / 2} / 8$.

In a harmonic trap, we have very similar equations with exactly the same structure. However, the dimensionless number density is now given by, $\tilde{n}=1 /\left(3 \tau^{3}\right)$. The function $n(z)$ and $f(z)$ shall take the form,

$$
\begin{aligned}
& n(z) \equiv \frac{1}{2} \int_{0}^{\infty} \frac{z e^{-t}}{\left(1+z e^{-t}\right)} t^{2} d t, \\
& f(z) \equiv \frac{1}{2} \int_{0}^{\infty} \ln \left(1+z e^{-t}\right) t^{2} d t,
\end{aligned}
$$

respectively. Finally, the constant $A$ in free energy should be read as $A=3 \tau^{4}$.

\section{RESULTS AND DISCUSSIONS}

We discuss separately the high-temperature free energy of a unitary Fermi gas in homogeneous space and in a harmonic trap.

\section{A. Free energy of a homogeneous unitary Fermi gas}

Fig. 1 presents the virial expansion prediction for the free energy as a function of temperature, for a balanced unitary Fermi gas or for a spin imbalanced system. For comparison, we show also the free energy of an ideal, non-interacting Fermi gas, $F^{(1)}$. To illustrate clearly the interaction effect, we show in Figs. $1 \mathrm{~b}$ and $1 \mathrm{c}$ the residue free energy or the interaction energy $\Delta F=F-F^{(1)}$, by subtracting the non-interacting background $F^{(1)}$. As shown in Fig. 1a and in the lower part of Fig. 1b for a balanced Fermi gas, in the presence of attractive interactions the second- and third-order virial expansion predict quantitatively the same free energy down to the Fermi degenerate temperature $T_{F}$. This suggests a very broad temperature window for applying the quantum virial expansion method. In case of repulsive interactions, however, the good agreement from the expansions at different order is found only at $T>5 T_{F}$, indicating that the virial expansion for a repulsively interacting Fermi gas is severely restricted. The similar applicability of virial expansion is observed as well for an imbalanced Fermi gas with a general spin imbalance $P$. On the other hand, by comparing the interaction energy at $P=0$ (Fig. 1b) and $P=0.5$ (Fig. 1c), a finite spin imbalance reduces the
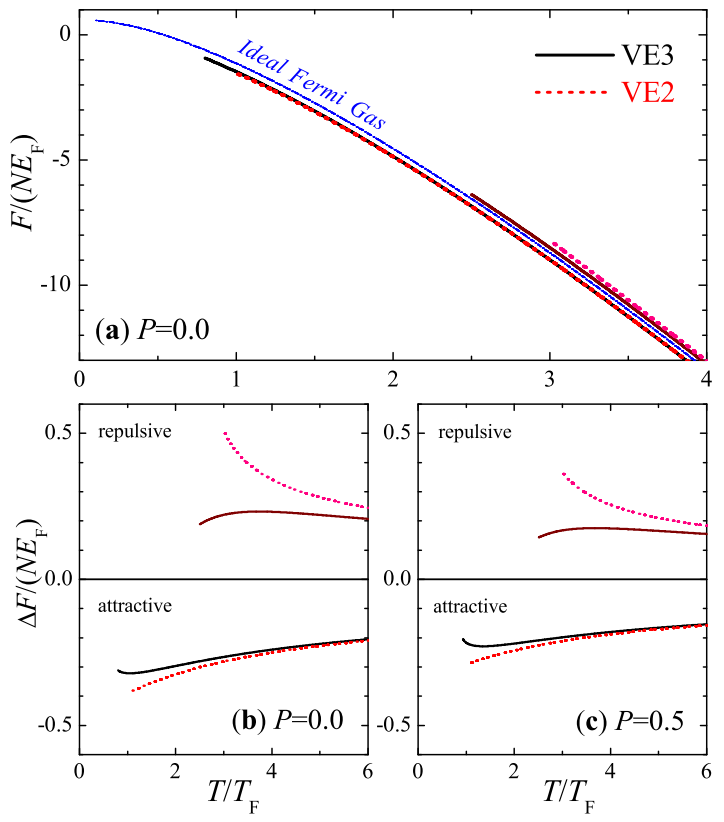

Figure 1: (color online) Temperature dependence of the free energy of a homogeneous unitary Fermi gas, calculated up to the second-order (dashed lines) or third-order (solid lines) using the quantum virial expansion theory. For comparison, we show the ideal, non-interacting free energy by a thin dotdashed line. As anticipated, the free energy of an attractive or a repulsive Fermi gas is always smaller or larger than that of an ideal Fermi gas, respectively.

interaction energy as expected, although the reduction is not so significant.

Fig. 2 reports the free energy as a function of the spin imbalance at a given temperature, for an attractive unitary Fermi gas (upper panel, Fig. 2a) or for a repulsive unitary Fermi gas (lower panel, Fig. 2b). The exact free energy would be bounded by the two successive expansion predictions and therefore could lie in between. With increasing the spin imbalance, the free energy of interacting Fermi gases approaches to the ideal, fully polarized limit. The reduction of interaction energy is clearly visible. For the case with repulsive interactions at $T=5 T_{F}$, where we believe the quantitative applicability of quantum virial expansion, the minimum of the free energy occurs at the balanced limit of $P=0$. This implies that there is no itinerant ferromagnetism at such a large temperature $5 T_{F}$. It therefore gives an upper bound for the ferromagnetic transition temperature of a homogeneous Fermi gas at unitarity.

\section{B. Free energy of a trapped unitary Fermi gas}

In analogy with the uniform free energy in Figs. 1 and 2, we show in Figs. 3 and 4, respectively, the dependence of the free energy on temperature and on spin imbalance. Due to the existence of harmonic traps and hence a factor 


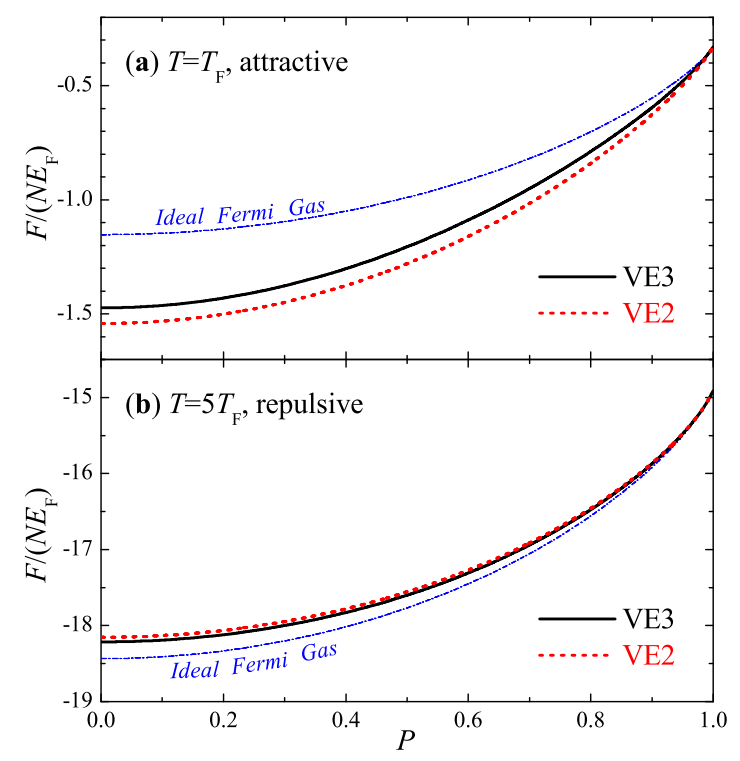

Figure 2: (color online) Free energy of a homogeneous unitary Fermi gas as a function of spin imbalance at a given temperature (as indicated), for attractive interactions or for repulsive interactions. The exact free energy could lie between the second- and third-order virial expansion predictions.

of $n^{-3 / 2}$ reduction for the $n$-th virial coefficient as shown in Eq. (23), the applicability of quantum virial expansion is greatly extended. At the third order, we estimate that the virial expansion is now quantitatively reliable down to $0.5 T_{F}$ for an attractive Fermi gas and down to $T_{F}$ for a repulsive Fermi gas.

We estimate also the upper bound for the ferromagnetic transition temperature of a trapped repulsively interacting Fermi gas at unitarity. As seen from Fig. 4b, the trapped free energy does not exhibit any signal for itinerant ferromagnetism at the Fermi degenerate temperature $T_{F}$. We thus conclude that $\left(T_{c}\right)_{\text {upper }}<T_{F}$ for a trapped unitary Fermi gas.

\section{Absence of itinerant ferromagnetism in the third order virial expansion}

The absence of itinerant ferromagnetism in our virial expansion may be analytically understood. To the third order, we calculate the two by two susceptibility matrix $\mathcal{S}=\left(\partial n_{\sigma} / \partial \mu_{\sigma^{\prime}}\right)$. A divergent or negative spin susceptibility $\chi_{S}=(\partial \delta n / \partial \delta \mu)$ in the balanced limit of $P=0$ would signal the instability to the formation of ferromagneitc order. The calculation of the susceptibility matrix and $\chi_{S}$ is straightforward. We find that for a homogeneous Fermi gas at unitarity,

$$
\mathcal{S}(P=0)=\frac{1}{k_{B} T \lambda^{3}}\left[\begin{array}{ll}
A & B \\
B & A
\end{array}\right]
$$

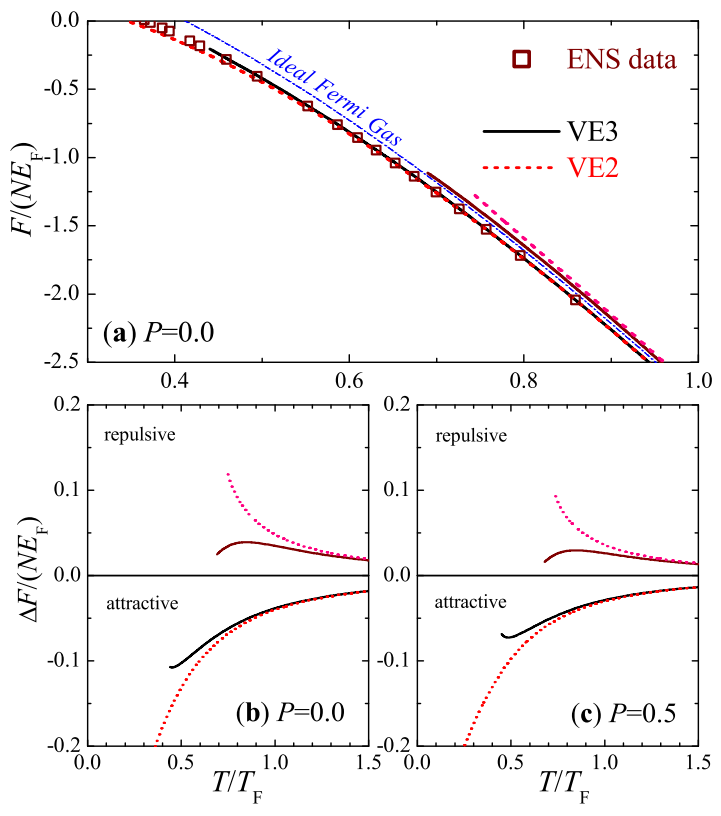

Figure 3: (color online) Temperature dependence of the free energy of a trapped unitary Fermi gas. We use the same notations as in Fig. 1. The experimental data for an attractive trapped Fermi gas at unitarity, reported by Salomon group at ENS [20], are shown by squares.

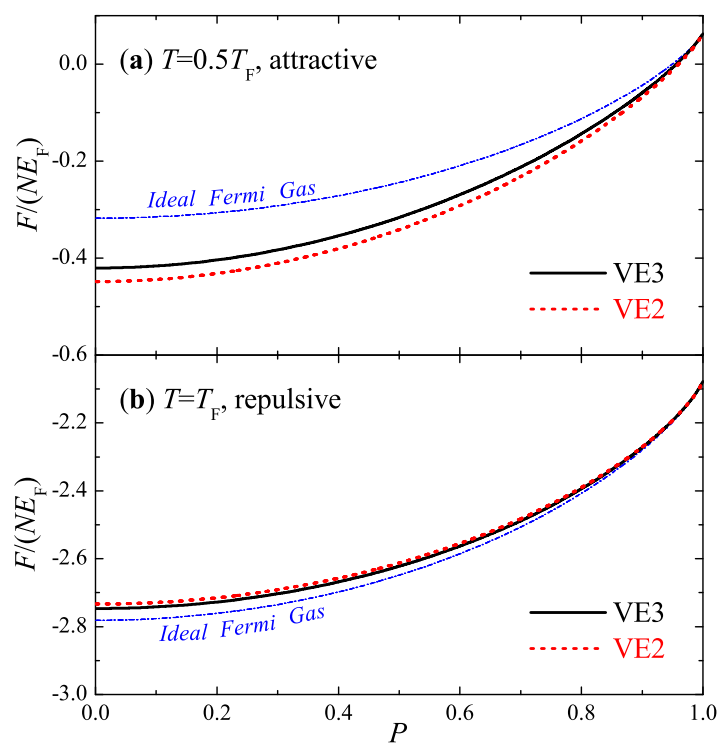

Figure 4: (color online) Free energy of a trapped unitary Fermi gas as a function of spin imbalance at a given temperature as indicated. The same notations as in Fig. 2 have been used.

where

$$
A=\frac{2}{\sqrt{\pi}} \int_{0}^{\infty} \frac{\sqrt{t} z e^{-t}}{\left(1+z e^{-t}\right)^{2}} d t+2 z^{2} \Delta b_{2, H}+5 z^{3} \Delta b_{3, H}
$$




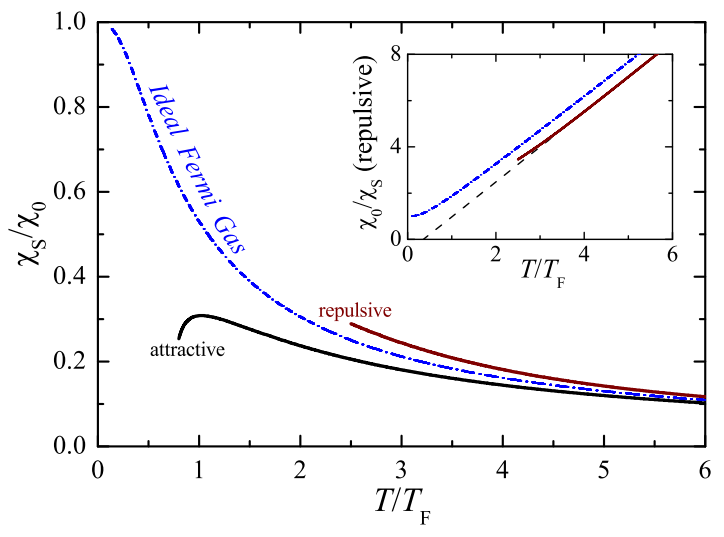

Figure 5: (color online) Spin susceptibility of a homogeneous Fermi gas at unitarity, normalized by the $T=0$ ideal Fermi gas susceptibility value $\chi_{0}=3 n /\left(2 \epsilon_{F}\right)$, with $n$ the density and $\epsilon_{F}$ the Fermi energy. The spin susceptibility of a repulsive or attractive Fermi gas at unitarity lies above or below the ideal Fermi Gas result (dot-dashed line). The inset shows the inverse of the spin susceptibility of a repulsive unitary Fermi gas. A linear extrapolation of the high- $T$ virial expansion result at $T / T_{F}=4 \sim 6$ to low temperatures indicates a ferromagnetic transition temperature of $T_{c} \sim 0.4 T_{F}$.

and

$$
B=2 z^{2} \Delta b_{2, H}+4 z^{3} \Delta b_{3, H} .
$$

The spin susceptibility $\chi_{S}=2(A-B) /\left(k_{B} T \lambda^{3}\right)$ is then given by,

$$
\chi_{S}=\frac{2}{k_{B} T \lambda^{3}}\left[\frac{2}{\sqrt{\pi}} \int_{0}^{\infty} \frac{\sqrt{t} z e^{-t}}{\left(1+z e^{-t}\right)^{2}} d t+z^{3} \Delta b_{3, H}\right] .
$$

It is easy to see that, because of a positive third virial coefficient $\Delta b_{3, H}^{r e p}$, the spin susceptibility of a repulsive unitary Fermi gas would always be finite and positive, for a finite fugacity. Therefore, there must be no ferromagnetic transition if we restrict the quantum virial expansion up to the third order. The same observation is found for a trapped repulsive Fermi gas at unitarity.

\section{Third order expansion of the spin susceptibility for a homogeneous Fermi gas at unitarity}

Figure 5 reports the numerical result of Eq. (33) for a homogeneous repulsive and attractive Fermi gas at unitarity, where the fugacity $z$ is solved consistently to the third order expansion in the number equation.

The spin susceptibility of a repulsive unitary Fermi gas is larger than that of an ideal, non-interacting Fermi gas, as expected. Accordingly, its inverse $\chi_{0} / \chi_{S}$ is smaller (see the inset). This might be a high-temperature indication of the ferromagetic instability. The transition temperature is conveniently determined from the condition $\chi_{S}^{-1}\left(T_{c}\right)=0$. By linearly extraplating the hightemperature result of $\chi_{S}^{-1}$ (in the window of $T=4 T_{F} \sim$ $\left.6 T_{F}\right)$ to low temperatures, we estimate very roughly that $T_{c} \sim 0.4 T_{F}$.

The spin susceptibility of an attractive unitary Fermi gas shown in Fig. 5 is also of interest. At finite temperatures, it is related to the measurement of the thermal spin flucutations:

$$
\frac{\triangle\left(N_{\uparrow}-N_{\downarrow}\right)^{2}}{N}=k_{B} T \frac{\chi_{S}}{n} .
$$

A shot noise measurement of the spin fluctuations therefore could be used as a sensitive thermometry for strongly interacting Fermi gases [35], provided that the spin susceptibility is known. This seems to be now accessible, since the shot noise measurements of the density fluctuations in a weakly interacting Fermi gas have already been demonstrated very recently [36, 37]. We find that the spin susceptibility of an attractive Fermi gas at unitarity is strongly suppressed with respect to the ideal Fermi gas result, even well above the degenerate temperature $T_{F}$. At $T=T_{F}$, the reduction is about $40 \%$.

\section{CONCLUSIONS AND REMARKS}

In conclusions, we have presented a quantum virial expansion theory for the thermodynamics of strongly interacting, spin-population imbalanced Fermi gases. The (imbalanced) virial coefficients are calculable through the cluster partition function with different spin configurations. We have shown the virial expansion of thermodynamics up to the third order and have discussed in detail the numerical results for the high-temperature free energy of a homogeneous or trapped Fermi gas at unitarity. The predicted free energy may confront future high-precision thermodynamic measurements.

An interesting application of our virial expansion theory is the determination of an upper bound for the ferromagnetic transition temperature of a repulsive Fermi gas at unitarity. We have proven numerically and analytically that, up to the third order quantum virial expansion fails to predict itinerant ferromagnetism. As the third-order virial expansion is quantitatively applicable at $T>T_{F}$ for a trapped repulsively interacting Fermi gas at unitarity, we therefore have estimated an upper bound $\left(T_{c}\right)_{\text {upper }}=T_{F}$ for the critical ferromagnetic temperature in harmonic traps. Further improvement of the upper bound requires the calculation of higher-order (imbalanced) virial coefficients, by using few-fermion solutions as the input [38 40].

Much wider applications of the quantum virial expansion method for strongly interacting quantum gases seem to be feasible. In the near future, we are interested in investigating the virial expansion of the Tan's contact [41, 42], the three-body recombination rate [43, 44], and the quantum viscosity [45, 46]. 


\section{Acknowledgments}

This work was supported in part by the ARC Centres of Excellence for Quantum-Atom Optics (ACQAO), ARC
Discovery Project No. DP0984522 and No. DP0984637, NSFC Grant No. 10774190, and NFRPC Grant No. 2006CB921404 and No. 2006CB921306.
[1] I. Bloch, J. Dalibard and W. Zwerger, Rev. Mod. Phys. 80, 885 (2008).

[2] S. Giorgini, L. P. Pitaevskii, and S. Stringari, Rev. Mod. Phys. 80, 1215 (2008).

[3] L. Radzihovsky and D. E. Sheehy, Rep. Prog. Phys. 73, 076501 (2010).

[4] F. Chevy and C. Mora, arXiv:1003.0801 (2010).

[5] P. Fulde and R. A. Ferrell, Phys. Rev. 135, A500 (1964).

[6] A. I. Larkin and Y. N. Ovchinnikov, Sov. Phys. JETP 20, 762 (1965).

[7] G. Orso, Phys. Rev. Lett. 98, 070402 (2007).

[8] H. Hu, X.-J. Liu, and P. D. Drummond, Phys. Rev. Lett. 98, 070403 (2007).

[9] X. W. Guan, M. T. Batchelor, C. Lee, and M. Bortz, Phys. Rev. B 76, 085120 (2007).

[10] X.-J. Liu, H. Hu, and P. D. Drummond, Phys. Rev. A 76, 043605 (2007).

[11] X.-J. Liu, H. Hu, and P. D. Drummond, Phys. Rev. A 78, 023601 (2008).

[12] Y.-A. Liao, A. S. C. Rittner, T. Paprotta, W. Li, G. B. Partridge, R. G. Hulet, S. K. Baur, and E. J. Mueller, arXiv:0912.0092 (2010); Nature, in press.

[13] T.-L. Ho and E. J. Mueller, Phys. Rev. Lett. 92,160404 (2004).

[14] C. J. Horowitz and A. Schwenk, Phys. Lett. B 638, 153 (2006).

[15] X.-J. Liu, H. Hu, and P. D. Drummond, Phys. Rev. Lett. 102, 160401 (2009).

[16] X.-J. Liu, H. Hu, and P. D. Drummond, Phys. Rev. A 82, 023619 (2010).

[17] X.-J. Liu, H. Hu, and P. D. Drummond, Phys. Rev. B 82, 054524 (2010).

[18] H. Hu, X.-J. Liu, and P. D. Drummond, Phys. Rev. Lett. 104, 240407 (2010).

[19] H. Hu, X.-J. Liu, and P. D. Drummond, Phys. Rev. A 81, 033630 (2010).

[20] S. Nascimbène, N. Navon, K. Jiang, F. Chevy, and C. Salomon, Nature 463, 1057 (2010).

[21] P. F. Bedaque and G. Rupak, Phys. Rev. B 67, 174513 (2003).

[22] J. T. Stewart, J. P. Gaebler, and D. S. Jin, Nature 454, 744 (2008).

[23] J. P. Gaebler, J. T. Stewart, T. E. Drake, D. S. Jin, A. Perali, P. Pieri, and G. C. Strinati, Nature Phys. 6, 569 (2010).

[24] G. Veeravalli, E. D. Kuhnle, P. Dyke, and C. J. Vale,
Phys. Rev. Lett. 101, 250403 (2008).

[25] E. C. Stoner, Proc. R. Soc. London. Ser. A 165, 372 (1938).

[26] R. A. Duine and A. H. MacDonald, Phys. Rev. Lett. 95, 230403 (2005).

[27] J. L. LeBlanc, J. H. Thywissen, A. A. Burkov, and A. Paramekanti, Phys. Rev. A 80, 013607 (2009).

[28] G. B. Jo , Y. R. Lee, J. H. Choi, C. A. Christensen, T. H. Kim, J. H. Thywissen, D. E. Pritchard, and W. Ketterle, Science 325, 1521 (2009).

[29] X. Cui and H. Zhai, Phys. Rev. A 81, 041602(R) (2010).

[30] H. Dong, H. Hu, X.-J. Liu, and P. D. Drummond, Phys. Rev. A 82, 013627 (2010).

[31] C. J. Horowitz and A. Schwenk, Phys. Lett. B 642, 326 (2006).

[32] C. J. Horowitz and A. Schwenk, Nucl. Phys. A 766, 55 (2006).

[33] F. Werner and Y. Castin, Phys. Rev. Lett. 97, 15041 (2006).

[34] F. Werner and Y. Castin, Phys. Rev. A 74, 053604 (2006).

[35] Q. Zhou and T.-L. Ho, arXiv:0908.3015 (2009).

[36] T. Mueller, B. Zimmermann, J. Meineke, J.-P. Brantut, T. Esslinger, and H. Moritz, arXiv:1005.0302 (2010).

[37] C. Sanner, E. J. Su, A. Keshet, R. Gommers, Y. Shi, W. Huang, and W. Ketterle, arXiv:1005.1309 (2010).

[38] J. von Stecher, C. H. Greene, and D. Blume, Phys. Rev. A 77, 043619 (2008).

[39] D. Blume and K. M. Daily, Phys. Rev. A 80, 053626 (2009).

[40] K. M. Daily and D. Blume, Phys. Rev. A 81, 053615 (2010).

[41] S. Tan, Ann. Phys. (NY) 323, 2952 (2008).

[42] E. D. Kuhnle, H. Hu, X.-J. Liu, P. Dyke, M. Mark, P. D. Drummond, P. Hannaford, and C. J. Vale, Phys. Rev. Let. 105, 070402 (2010).

[43] B. D. Esry, C. H. Greene, and J. P. Burke, Jr., Phys. Rev. Lett. 83, 1751 (1999).

[44] P. F. Bedaque, E. Braaten, and H.-W. Hammer, Phys. Rev. Lett. 85, 908 (2000).

[45] E. Taylor and M. Randeria, Phys. Rev. A 81, 053610 (2010).

[46] T. Enss, R. Haussmann, and W. Zwerger, arXiv:1008.0007 (2010). 